\title{
Analysis of Vertical Dynamic Cross Interaction between Adjacent Footings for Railway Tracks through Soil
}

\author{
Jue WANG \\ College of Mechanical \& Electrical Engineering, Hohai University, Changzhou 213022, China
}

\begin{abstract}
An efficient semi-analytical method is presented for the vertical analysis of adjacent railway tracks considering the dynamic cross interaction (DCI) effect through the semi-infinite soil medium. The interfaces between soil and tracks are discretized into a series of strip elements to solve the unknown contact pressure influenced by DCI effect. The Green function for each element under uniform harmonic excitation is derived and calculated by the piecewise integration and Cauchy principal value integral. The vertical impedance matrix is finally obtained by the superposition method. The accuracy and the effectiveness in high frequency range are verified by the convergence study, as well as the comparison study with the rigorous results from the mix-boundary-value method. The influence of the soil properties and the tracks distance on the DCI effect are discussed in detail in the parameter studies. It is shown that the DCI effect increases with the decrease of the distance ratio $\mathrm{S} / \mathrm{L}$.
\end{abstract}

\section{Introduction}

Railway tracks are sometimes constructed close to each other due to the limitation of the space, as shown in Fig. 1. To accurately analyze the dynamic characteristics of the railway tracks, it is necessary to consider not only the interaction between soil and tracks but also the dynamic cross interaction (DCI) between adjacent tracks through soil medium. The vertical reactions of the soil against tracks can be described by frequency-dependent stiffness and damping coefficients which are defined as vertical impedances. Therefore, vertical impedances are key parameters to evaluate the DCI effect between the adjacent footings of railway tracks.

The approximate solution for a strip footing impedance was obtained through stress boundary-value method which was based on the assumptions of press distributions (static rigidity, uniform and parabolic) beneath the foundation [1]. Anam [2] assumed a combination of two specified distributions for the dynamic stiffness of massless strip surface foundations. However, apart from a single track footing, those assumed symmetrical distribution of contact pressures may not be valid for adjacent tracks. The more rigorous solutions for the strip footing are analyzed as a mixed boundary-value problem formulated in terms of the governing dual integral equations [3]. Ma et al. [4] transformed the dual integral equations into a set of linear equations based on an infinite series of orthogonal Jacobi polynomials to obtain the impedance of a rigid strip footing. It should be noted that there are limitations to obtain the contact pressure functions for adjacent tracks considering DCI as the solutions of dual integral equations cannot be expressed by elementary functions.
Therefore, the analyses of dynamic interactions between multiple tracks are mainly through numerical methods such as the finite element method and the boundary element method since the rapid progress in computer technique $[5,6]$. However, these methods are far too time-consuming due to the semi-infinite soil medium. The analytical method for the vertical impedance of adjacent tracks based on the elastic half-space theory is still very rare [7, 8], while it is efficient and has great significance for solving the seismic response of railway tracks and assessing structures safety.

In this paper an efficient semi-analytical approach is developed to obtain the vertical impedances of adjacent footings for railway tracks rested on a linear elastic halfspace with consideration of DCI effect. The half-space Green function for vertical uniform harmonic excitation is derived to determine the contact pressures at the interfaces between soil and footings. A superposition method is used to finally obtain the vertical impedances. The validity and wide applicability of the present method has been verified by the comparative studies. The DCI effect on vertical impedances of adjacent tracks is illustrated by the parametric studies.

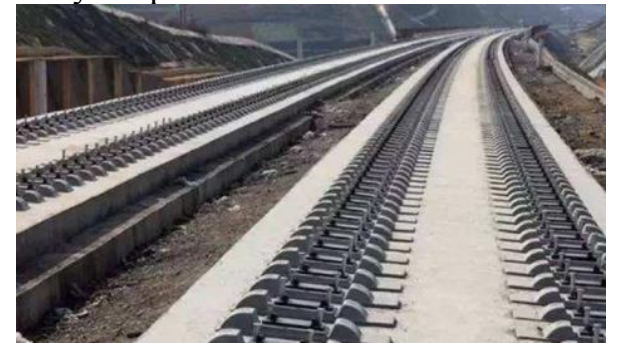

Fig. 1. Adjacent railway tracks in track engineering 


\section{$2 \mathrm{DCl}$ model for adjacent railway tracks}

As the railway tracks have a high ratio of length to width, it is reasonable to consider the problem as a generalized plane strain model. The vertical dynamic of adjacent railway tracks rested on an elastic semi-infinite soil medium with separation distance $S$ is considered as shown in Fig. 2. Track A with the width $L_{a}$ and Track B with the width $L_{b}$ are excited by the vertical excitation $Q_{a} e^{\mathrm{i} \omega t}$ and $Q_{b} e^{\mathrm{i} \omega t}$, respectively. Considering DCI effect, the dynamic force and deformation relationship of the adjacent tracks can be expressed by

$$
\left[\begin{array}{l}
Q_{a} \\
Q_{b}
\end{array}\right]=\left[\begin{array}{ll}
\mathfrak{R}_{a a} & \mathfrak{R}_{a b} \\
\mathfrak{R}_{b a} & \mathfrak{R}_{b b}
\end{array}\right]\left[\begin{array}{l}
W_{a} \\
W_{b}
\end{array}\right]
$$

where $W$ is the vertical displacement with subscripts $a$ and $b$ indicating the notations belong to corresponding tracks. The term $\mathrm{e}^{\mathrm{i} \omega t}$ is hereafter omitted from all displacements and forces for brevity. The complex elements $\mathfrak{R}$ in the matrix are impedances of the system. The real and imaginary part of $\mathfrak{R}$ can be interpreted as frequency-dependent springs with coefficient $K(\omega)$ and dashpots with coefficient $C(\omega)$ respectively, as shown in Fig. 3.

To determine the contact pressures between soil and tracks, the interfaces are discretized into $N$ and $M$ surface strip elements. It is assumed that the $n$-th element $(n=1, \ldots, N)$ beneath Track A is subjected to a uniform vertical pressure $q_{n}^{(a)}$. Similarly, the $m$-th element $(n=1, \ldots, M)$ beneath Track $\mathrm{A}$ is subjected to a uniform vertical pressure $q_{m}^{(b)}$.

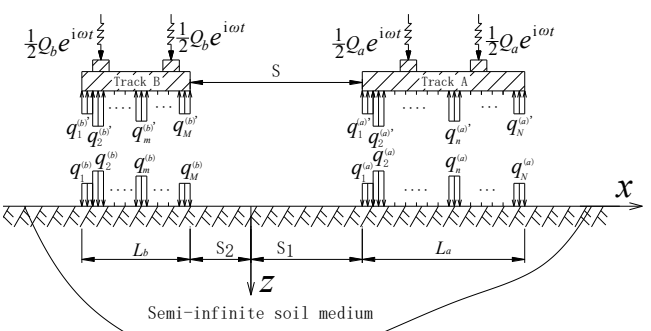

Fig. 2. Adjacent railway tracks rested on semi-infinite soil medium subjected to vertical harmonic excitations

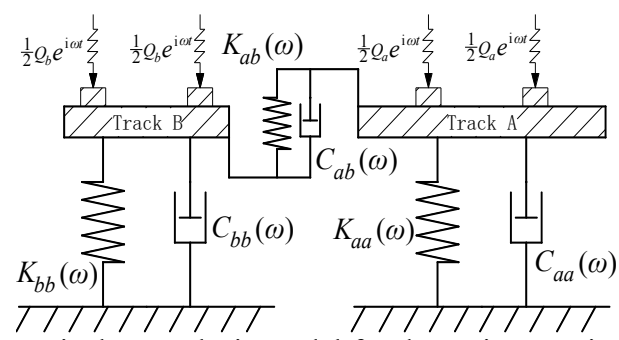

Fig. 3. Equivalent analysis model for dynamic cross interaction between adjacent railway tracks with springs and dashpots

\section{Formulations}

\subsection{Green function for vertical uniform harmonic excitation on half-space}

Based on the Cartesian coordinate system $(x-z)$ with $z=0$ at the free surface, the wave equations in an elastic halfspace composed of homogeneous and isotropic solid are given by

$$
\left\{\begin{array}{l}
\rho \frac{\partial^{2} u(x, z, t)}{\partial t^{2}}=(\lambda+G) \frac{\partial \bar{\varepsilon}}{\partial x}+G \nabla^{2} u(x, z, t) \\
\rho \frac{\partial^{2} w(x, z, t)}{\partial t^{2}}=(\lambda+G) \frac{\partial \bar{\varepsilon}}{\partial z}+G \nabla^{2} w(x, z, t)
\end{array}\right.
$$

where $\rho$ is the density of the elastic soil, $G$ and $\lambda$ are the elastic Lamé constants, $u$ and $w$ are the displacement components of the soil in $x$ and $z$ directions. The steadystate vertical dynamic of the soil satisfies $u=U \mathrm{e}^{\mathrm{i} \omega t}$ and $w=W \mathrm{e}^{\mathrm{i} \omega t} \cdot \bar{\varepsilon}=\frac{\partial u}{\partial x}+\frac{\partial w}{\partial z}$ and $\nabla^{2}=\left(\frac{\partial^{2}}{\partial x^{2}}+\frac{\partial^{2}}{\partial z^{2}}\right)$ denote the volumetric strain and the Laplace operator, respectively.

Considering the unit vertical uniform traction applied at the interval $\left[x_{1}, x_{2}\right]$, the boundary conditions at ground surface $z=0$ are given by

$$
\sigma_{z z}(x, 0)=\left\{\begin{array}{cc}
-q & a \leq x \leq b \\
0 & \text { others }
\end{array}, \tau_{x z}(x, 0)=0\right.
$$

The general solutions of Eq. (2) for a half-space can be obtained by the method of separation of variable. The general solutions for stresses $\sigma_{z z}$ and $\tau_{x z}$ can be obtained by the stress-displacement differential relationship. Based on the boundary condition of Eq. (3), the unknown coefficients in the general solutions can be determined. Therefore, the vertical displacement fields $w(x, z)$ due to the unit vertical uniform load at the surface of the soil medium can be obtained as

$$
\begin{aligned}
w(x, z)= & \frac{\mathrm{i} q}{2 \pi G} \int_{-\infty}^{\infty} \frac{-\alpha\left[\left(2 \xi^{2}-k^{2}\right) \exp (-\alpha z)+2 \xi^{2} \exp (-\beta z)\right]}{F(\xi) \xi} \\
& \left\{\mathrm{e}^{[\mathrm{i} \xi(x-\mathrm{a})]}-\mathrm{e}^{[\mathrm{i} \xi(x-\mathrm{b})]}\right\} d \xi
\end{aligned}
$$

where $F(\xi)=\left(2 \xi^{2}-k^{2}\right)^{2}-4 \xi^{2} \alpha \beta \quad ; \quad \alpha^{2}=\xi^{2}-h^{2} \quad$ and $\beta^{2}=\xi^{2}-k^{2}$ with $h=\sqrt{\omega / V_{\mathrm{p}}}$ and $k=\sqrt{\omega / V_{\mathrm{s}}} . \xi$ is the wave number; $V_{\mathrm{p}}=\sqrt{\lambda+2 G / \rho}$ is the dilatational wave velocity; $V_{\mathrm{s}}=\sqrt{G / \rho}$ is the shear wave velocity.

\subsection{Vertical impedance matrix of adjacent footings for railway tracks}

Following the superposition approach, the vertical displacement $W(x, 0)$ at any point of the soil surface caused by a series of strip elements beneath the Track A and Track B can be obtained from Eq. (4) as follows

$$
\begin{aligned}
& w(x, 0)=\frac{-1}{\pi G}\left\{\sum _ { n = 1 } ^ { N } q _ { n } ^ { ( a ) } \int _ { 0 } ^ { \infty } \frac { L _ { a } \sqrt { p ^ { 2 } - \vartheta ^ { 2 } } } { 2 F ( p ) p a _ { 0 } } \left\{\operatorname { s i n } \left[p \frac{2 a_{0}}{L_{a}}\left(x-\left(S_{1}+\frac{(n-1) L_{a}}{N}\right)\right]\right.\right.\right. \\
& \left.-\sin \left[p \frac{2 a_{0}}{L_{a}}\left(x-\left(S_{1}+\frac{n L_{a}}{N}\right)\right)\right]\right\} d p+\sum_{m=1}^{M} q_{n}^{(b)} \int_{0}^{\infty} \frac{L_{a} \sqrt{p^{2}-\vartheta^{2}}}{2 F(p) p a_{0}}\left\{\sin \left[p \frac{2 a_{0}}{L_{a}}\left(x-\left(-\left(L_{b}+S_{2}\right)+\frac{(m-1) L_{b}}{M}\right)\right)\right]\right. \\
& \left.\left.-\sin \left[p \frac{2 a_{0}}{A}\left(x-\left(-\left(L_{b}+S_{2}\right)+\frac{m L_{b}}{M}\right)\right)\right]\right\} d p\right\}
\end{aligned}
$$

in which, $\quad F(p)=\left(2 p^{2}-1\right)^{2}-4 p^{2} \sqrt{p^{2}-\vartheta^{2}} \sqrt{p^{2}-1}$, $a_{0}=\omega L_{a} /\left(2 V_{s}\right), \xi=p k$ and $\vartheta=h / k$. 
The following flexibility equation of the system can be established by substituting the centrol coordintae of each element into Eq. (5) as follows

$$
\left[\begin{array}{ll}
{\left[\boldsymbol{A}_{i j}^{(a)}\right]_{N \times N}} & {\left[\boldsymbol{B}_{i j}^{(a)}\right]_{N \times M}} \\
{\left[\boldsymbol{A}_{i j}^{(b)}\right]_{M \times N}} & {\left[\boldsymbol{B}_{i j}^{(b)}\right]_{M \times M}}
\end{array}\right]\left\{\begin{array}{l}
\boldsymbol{q}^{(a)} \\
\boldsymbol{q}^{(b)}
\end{array}\right\}=\left\{\begin{array}{l}
\boldsymbol{w}^{(a)} \\
\boldsymbol{w}^{(b)}
\end{array}\right\}
$$

in which,

$$
\begin{aligned}
A_{i j}^{(a)}= & -\frac{1}{\pi G} \int_{0}^{\infty} \frac{L_{a} \sqrt{p^{2}-\vartheta^{2}}}{2 F(p) p a_{0}}\left\{\sin \left[p \frac{2 a_{0}}{L_{a}}\left(\frac{(i-j) L_{a}}{N}+\frac{L_{a}}{2 N}\right)\right]\right. \\
& \left.-\sin \left[p \frac{2 a_{0}}{L_{a}}\left(\frac{(i-j) L_{a}}{N}-\frac{L_{a}}{2 N}\right)\right]\right\} d p \\
B_{i j}^{(a)}= & -\frac{1}{\pi G} \int_{0}^{\infty} \frac{L_{a} \sqrt{p^{2}-\theta^{2}}}{2 F(p) p a_{0}}\left\{\sin \left[p \frac{2 a_{0}}{L_{a}}\left(\left(S+L_{b}\right)+\frac{(2 i-1) L_{a}}{2 N}-\frac{(j-1) L_{b}}{M}\right)\right]\right. \\
& \left.-\sin \left[p \frac{2 a_{0}}{b}\left(\left(S+L_{b}\right)+\frac{(2 i-1) L_{a}}{2 N}-\frac{j L_{b}}{M}\right)\right]\right\} d p \\
A_{i j}^{(b)}= & -\frac{1}{\pi G} \int_{0}^{\infty} \frac{L_{a} \sqrt{p^{2}-\theta^{2}}}{2 F(p) p a_{0}}\left\{\sin \left[p \frac{2 a_{0}}{L_{a}}\left(-\left(S+L_{b}\right)+\frac{(2 i-1) L_{b}}{2 M}-\frac{(j-1) L_{a}}{N}\right)\right]\right. \\
& \left.-\sin \left[p \frac{2 a_{0}}{L_{a}}\left(-\left(S+L_{b}\right)+\frac{(2 i-1) L_{b}}{2 M}-\frac{j L_{a}}{N}\right)\right]\right\} d p \\
B_{i j}^{(b)} & =-\frac{1}{\pi G} \int_{0}^{\infty} \frac{L_{a} \sqrt{p^{2}-\theta^{2}}}{2 F(p) p a_{0}}\left\{\sin \left[p \frac{2 a_{0}}{L_{a}}\left(\frac{(i-j) L_{b}}{M}+\frac{L_{b}}{2 M}\right)\right]\right. \\
& \left.-\sin \left[p \frac{2 a_{0}}{L_{a}}\left(\frac{(i-j) L_{b}}{M}-\frac{L_{b}}{2 M}\right)\right]\right\} d p
\end{aligned}
$$

Eqs. (7a-7d) are multi-value improper integral which can be precisely calculated by the piecewise integration and the Cauchy principal value integral ${ }^{[9]}$. For example Eq. (7a) can be expressed as follows

$$
A_{i j}^{(a)}=\frac{1}{2 \pi G}\left(f_{1}+\mathrm{i} f_{2}\right)
$$

in which,

$$
\begin{gathered}
f_{1}=-\int_{\vartheta}^{1} \frac{L_{a} \sqrt{p^{2}-\vartheta^{2}}\left(2 p^{2}-1\right)^{2}}{\left[\left(2 p^{2}-1\right)^{4}-16 p^{4}\left(p^{2}-\vartheta^{2}\right)\left(p^{2}-1\right)\right] p a_{0}} G(p) d p \\
-\overline{\mathrm{P}} \int_{1}^{\infty} \frac{L_{a} \sqrt{p^{2}-\vartheta^{2}}}{\left[\left(2 p^{2}-1\right)^{2}-4 p^{2} \sqrt{p^{2}-\vartheta^{2}} \sqrt{p^{2}-1}\right] p a_{0}} G(p) d p \\
f_{2}=-\int_{0}^{\vartheta} \frac{L_{a} \sqrt{\vartheta^{2}-p^{2}}}{\left[\left(2 p^{2}-1\right)^{2}+4 p^{2} \sqrt{\vartheta^{2}-p^{2}} \sqrt{1-p^{2}}\right] p a_{0}} G(p) d p \\
-\int_{\vartheta}^{1} \frac{4 L_{a} p^{2}\left(p^{2}-\vartheta^{2}\right) \sqrt{1-p^{2}}}{\left[\left(2 p^{2}-1\right)^{4}-16 p^{4}\left(p^{2}-\vartheta^{2}\right)\left(p^{2}-1\right)\right] p a_{0}} G(p) d p+\pi \cdot \frac{L_{a} \sqrt{\kappa^{2}-\vartheta^{2}}}{\left.[F(p) p]\right|_{p=\kappa} a_{0}} G(\kappa)
\end{gathered}
$$

with $G(p)=\sin \left[p \frac{2 a_{0}}{L_{a}}\left(\frac{(i-j) L_{a}}{N}+\frac{L_{a}}{2 N}\right)\right]-\sin \left[p \frac{2 a_{0}}{L_{a}}\left(\frac{(i-j) L_{a}}{N}-\frac{L_{a}}{2 N}\right)\right]$.

$\kappa$ is the root of $F(p)$ and $\bar{P}$ means the Cauchy principal value integral.

When the strip elements beneath the tracks satisfy $\boldsymbol{w}^{(a)}=\{1\}, \quad \boldsymbol{w}^{(b)}=\{0\}, \quad$ the unknown coefficients $q_{1}^{(a)} \cdots q_{N}^{(a)}, q_{1}^{(b)} \cdots q_{M}^{(b)}$ can be determined by the solution of Eq. (6). The impedance element $\mathfrak{R}_{a a}$ and $\mathfrak{R}_{b a}$ of impedance matrix in Eq. (1) can be obtained by

$$
\begin{aligned}
& \mathfrak{R}_{a a}=\frac{L_{a}}{N} \sum_{n=1}^{N} q_{n}^{(a)}=K_{a a}+\mathrm{i} C_{a a} \\
& \mathfrak{R}_{b a}=\frac{L_{b}}{M} \sum_{m=1}^{M} q_{m}^{(b)}=K_{b a}+\mathrm{i} C_{b a}
\end{aligned}
$$

When the strip elements beneath the tracks satisfy $\boldsymbol{w}^{(a)}=\{0\}, \quad \boldsymbol{w}^{(b)}=\{1\}, \quad$ the unknown coefficients $q_{1}^{(a)} \cdots q_{N}^{(a)}, q_{1}^{(b)} \cdots q_{M}^{(b)}$ can be determined by the solution of Eq. (6). The impedance element $\mathfrak{R}_{b b}$ and $\mathfrak{R}_{a b}$ of impedance matrix in Eq. (1) can be obtained by

$$
\begin{aligned}
& \mathfrak{R}_{b b}=\frac{L_{b}}{M} \sum_{m=1}^{M} q_{m}^{(b)}=K_{b b}+\mathrm{i} C_{b b} \\
& \mathfrak{R}_{a b}=\frac{L_{a}}{N} \sum_{n=1}^{N} q_{n}^{(a)}=K_{a b}+\mathrm{i} C_{a b}
\end{aligned}
$$

\section{Numerical Examples}

\subsection{Comparison studies}

The flexibility of a rigid strip footing was obtained by Luco [3] based on the mixed boundary-value approach with Fredholm integral equations. As considerable difficulties in solving Fredholm integral equations numerically or analytically, therefore only a special case for Poisson's ratio $v=0.5$ was studied rigorously in Luco's solution. The cases with Poisson's ratio $v<0.5$ were approximately studied by using the dominant part of the singular integral equation to evaluate the impedances of the strip footing, which is only valid for the low frequency $a_{0} \leq 1$. In order to compare with the Luco's solutions, the impedance $\mathfrak{R}$ is transformed into the flexibility by $F=\mathfrak{R}^{-1}$. The flexibility of the footing with respect to dimensionless exciting frequency is plotted in Fig. 4 for three different Poisson's ratios $v=0.5,1 / 3,0.25$. In Fig. 4, the maximum relative errors between two solutions are less than $10 \%$. The agreement with Luco's solution approves the correctness and effectiveness of the present method. In addition, Fig. 4(b) shows that the present method covers a wider range of frequency than Luco's method.

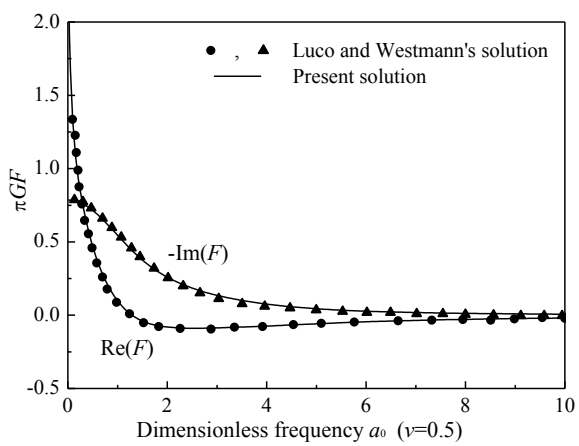

(a) $v=0.5$

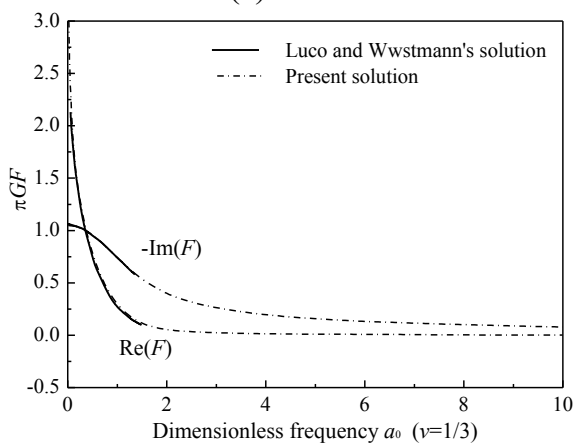

(b) $v=1 / 3$

Fig. 4. Vertical flexibility $F\left(a_{0}\right)$ of a strip footing

4.2 Vertical dynamic contact pressures at the interfaces 
The dynamic contact pressures beneath the tracks are compared to that of a single one to reveal the significance of DCI effect as shown in Fig. 5. Two dimensionless frequencies $a_{0}=0.2,3$ for the vertical excitations are considered. The distance ratio $S / L=0.25$ and the Poisson's ratio $v=1 / 3$ are considered in the analysis. $\overline{\boldsymbol{x}}$ is defined as the local coordinate with the origin at the center of each track. The non-dimensional contact pressure is $\bar{q}$ $=L q /\left(K_{\text {sig }} H\right)$ where $K_{\text {sig }}$ is the dynamic stiffness of the single track and $H$ is its displacement amplitude. It can be seen from Fig. 5 that adjacent tracks present a skewed distribution of the contact pressure due to DCI effect. Therefore, the assumption of the symmetric stress distributions such as the static rigid distribution assumption for a single footing will induce a considerable error in the dynamic analysis of adjacent tracks. Moreover, comparison between Fig. 5a and Fig. 5b indicates that the shape and magnitude of the contact pressures are quite sensitive to the variation of the dimensionless frequency $a_{0}$.

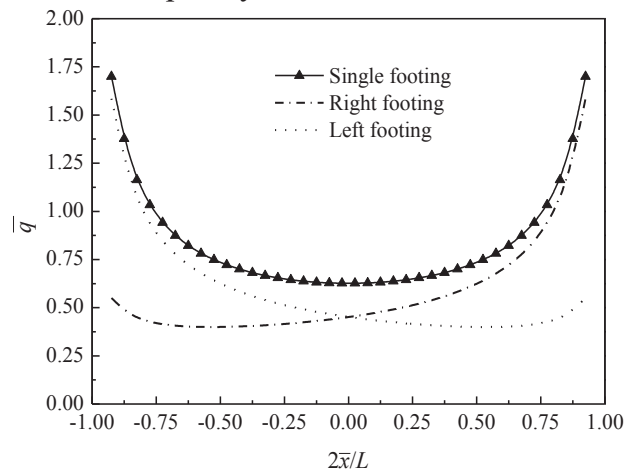

(a) $a_{0}=0.2$

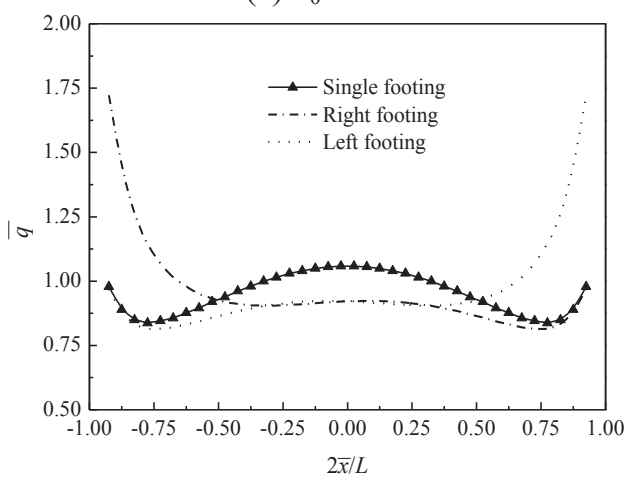

(a) $a_{0}=3.0$

Fig. 5. DCI effect between adjacent tracks on dynamic contact pressures

\subsection{Influence of distance ratio on $\mathrm{DCl}$ effect}

Two identical tracks with the same width $L$, separated by a distance $S$ and subjected to vertical harmonic excitation, are used to investigate the influence of the distance ratio $S / L$ on DCI effect. The vertical flexibility $F_{a a}$ of a footing considering DCI effect with respect to the dimensionless exciting frequency $a_{0}$ for different distance ratios $(S / L=0.125,0.5,4.0, \infty)$ are displayed in Figs. 6 for $v=0.25$ and Fig. 7 for $v=1 / 3$. It can be seen that the flexibility for two footings in the case of a closely spaced distance ratio such as the distance ratio $S / L=0.125$ fluctuates obviously around that of the single footing.
However, the DCI effect decreases with the increase of the distance ratio. In addition, the comparison between Fig. 6 and Fig. 7 shows that Poisson's ratio of the soil has little effect on DCI effect.

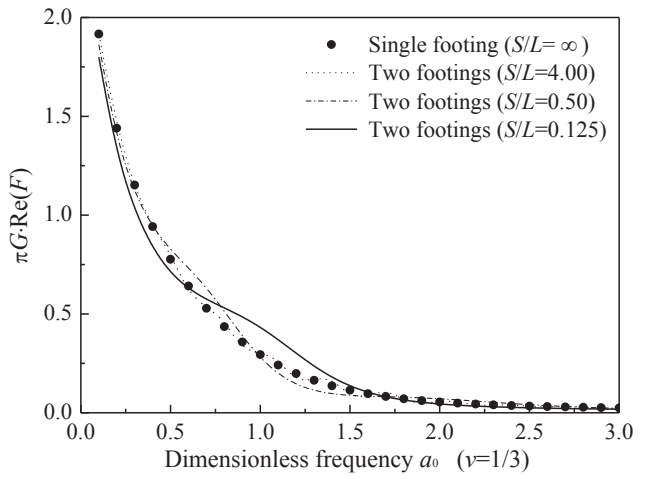

(a) Real part

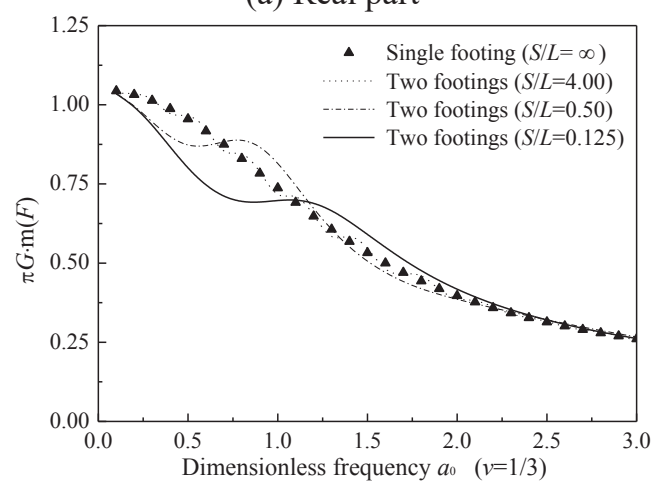

(b) Imaginary part

Fig. 6. Vertical flexibility $F_{a a}\left(a_{0}\right)$ in consideration of DCI effect for $v=1 / 3$

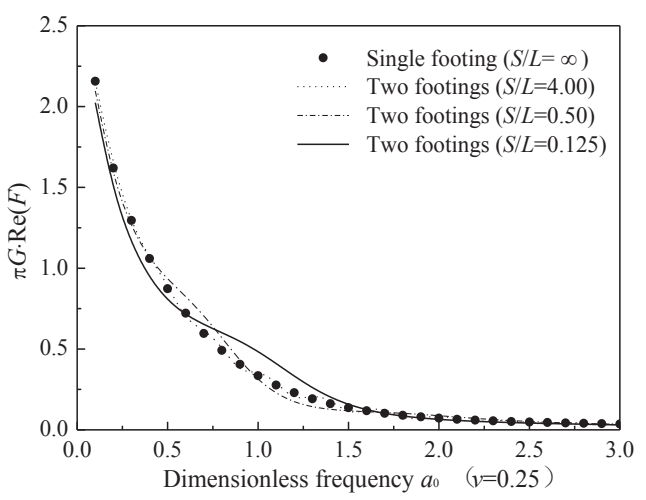

(a) Real part

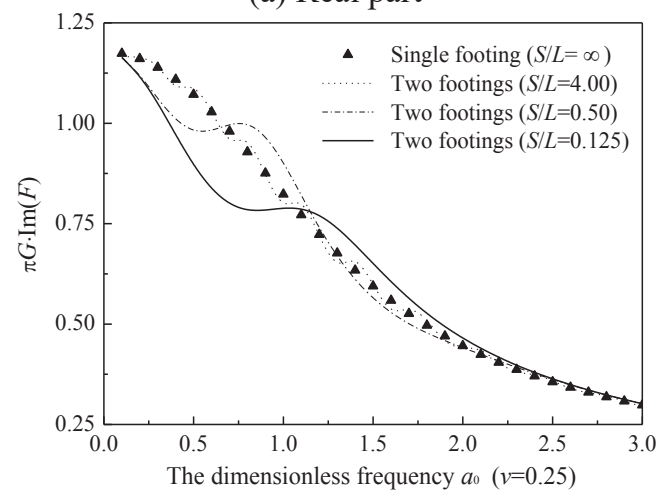

(b) Imaginary part

Fig. 7. Vertical flexibility $F_{a a}\left(a_{0}\right)$ in consideration of DCI effect for $v=0.25$ 


\section{Conclusions}

An efficient semi-analytical method has been developed to analysis the vertical dynamic of adjacent railway tracks considering DCI effect. The approach is of significance for dynamic analysis and seismic design of rail tracks with close space. The dynamic contact pressures and impedances have been studied in detail and following conclusions can be emphasized:

(1) The present method overcomes the mathematical limitations in the mixed boundary-value method and can provide the dynamic impedances of adjacent railway tracks within a wide scope of excitation frequency. The computational stability and accuracy of the present method have been verified by the convergence studies and the comparison examples.

(2) The distributions of contact pressures at the interface between the soil and tracks are influenced by the frequency of excitation and DCI effect. Therefore, those symmetric distribution assumptions for a single footing would induce a considerable error in DCI analysis.

(3) The DCI effect increases with the decrease of distance ratio $S / L$ between adjacent footings for tracks. The impedance of the track considering DCI effect fluctuates around that of a single one when the distance ratio becomes small.

\section{Acknowledgments}

The financial supports from the National Natural Science Foundation of China (51708179), Natural Science Foundation of Jiangsu Province (BK20170299), China Postdoctoral Science Foundation (2018M632216) and Fundamental Research Funds for the Central Universities (2016B15014), are greatly acknowledged.

\section{References}

[1] Sung T Y, Vibration in Semi-infinite Solids due to Periodic Surface Loadings Symposium of Dynamic Testing of Soils 156: 35-64 (1953).

[2] Anam J, Roësset M, Dynamic stiffnesses of surface foundations: an explicit solution Int. J. Geomech 4: 216-223 (2004).

[3] Luco J E Westmann R A, Dynamic response of a rigid footing bonded to an elastic half-space J Eng. Mech. Div. ASME 39:527-534 (1972).

[4] Ma X H, Cheng Y M, Au S K, Rocking vibration of a rigid strip footing on saturated soil Comput. Geotech. 36: 928-933 (2009).

[5] Padron L A, Aznarez J J, Maeso O, Dynamic structure-soil-structure interaction between nearby piled buildi ngs under seismic excitation by BEMFEM model Soil Dyn. Earthq. Eng. 29:1084-1096 (2009).

[6] Ghosh P, FLAC based numerical studies on dynamic interference of two nearby embedded machine foundations Geotech. Geol. Eng. 30:1161-1181 (2012).

[7] Wang Y, Rajapakse R, Shah A H, Dynamic interaction between flexible strip foundations Earthq. Eng. Struct. Dyn. 20: 441-454 (1991).

[8] Wang J, Lo S H, Zhou D et al. Frequency-dependent impedance of a strip foundation group and its representation in time domain App. Math. Model. 39: 2861-2881 (2015).

[9] Philip J D Philip R, Methods of Numerical Integration: Second Edition New York: Dover Publications (2007). 